\title{
Ferroelectrics
}

\section{Ferroelectric based fractal phononic crystals: wave propagation and band structure}

\author{
Selami Palaz, Zafer Ozer, Amirullah M. Mamedov \& Ekmel Ozbay
}

To cite this article: Selami Palaz, Zafer Ozer, Amirullah M. Mamedov \& Ekmel Ozbay (2020)

Ferroelectric based fractal phononic crystals: wave propagation and band structure, Ferroelectrics, 557:1, 85-91, DOI: 10.1080/00150193.2020.1713352

To link to this article: https://doi.org/10.1080/00150193.2020.1713352

\section{Published online: 07 Apr 2020.}

Submit your article to this journal ๘

Lll Article views: 47

Q View related articles $\asymp$

View Crossmark data $\nearrow$ 


\title{
Ferroelectric based fractal phononic crystals: wave propagation and band structure
}

\author{
Selami Palaz ${ }^{\mathrm{a}}$, Zafer Ozer ${ }^{\mathrm{b}}$, Amirullah M. Mamedov ${ }^{\mathrm{c}, \mathrm{d}}$, and Ekmel Ozbay ${ }^{\mathrm{c}}$ \\ ${ }^{a}$ Faculty of Sciences and Letters, Department of Physics, Harran University, Sanliurfa, Turkey; \\ ${ }^{b}$ Department of Electronic and Automation, Mersin Vocational High School, Mersin University, Mersin,

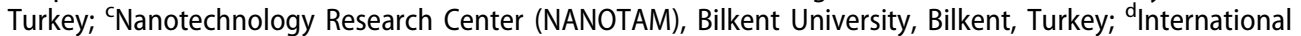 \\ Scientific Center, Baku State University, Baku, Azerbaijan
}

\begin{abstract}
In this study, the band structure and transmission in multiferroic based Sierpinski carpet phononic crystal are investigated based on finite element simulation. In order to obtain the band structure of the phononic crystal $(\mathrm{PnC})$, the Floquet periodicity conditions were applied to the sides of the unit cell. The square lattice PnC consists of various piezoelectric inclusion in a rubber matrix with square and circular cross section.
\end{abstract}

\section{ARTICLE HISTORY}

Received 14 July 2019

Accepted 24 December 2019

\section{KEYWORDS}

Phononic crystals;

ferroelectric; fractal; band

structure; finite

element method

\section{Introduction}

Fractals have repetitive and infinitely long-lasting shapes from inward to outward direction. Fractals contain elements such as ratio, balance and harmony, and they have attracted the interest of researchers [1-3]. Fractal designs are an innovative approach to discover new types of Photonic crystals [4-6]. Studies have been performed to increase the band gap of PnC using structures in different fractal designs [7-12]. The Sierpinski Carpet, as one of the fractal designs, was first described by Wacław Sierpiński in 1916 [1].

In this study, quasi-Sierpinski carpet Phononic crystals were first identified as unit cells and the band structure obtained in the direction of the $\Gamma-\mathrm{X}-\mathrm{M}-\Gamma$. Figure 1 shows the unit cells of different filling fractions of traditional Sierpinski-carpet phononic crystals.

\section{Materials and method}

The Floquet periodicity conditions were applied to the sides of unit cells 1-4 and 2-3 in Figure 1a in order to obtain the band structure of the PnC. The square lattice PnC consists of $\mathrm{LiTaO}_{3}$ inclusions in a rubber matrix with a square and circular cross sections.

The square unit cell with an "a" edge length is divided into 9 equal subframes according to the traditional Sierpinski carpet production method, and the Square in the middle is filled with a piezoelectric rod to form the first step $(\mathrm{L}=1)$. " $\mathrm{L}=2$ " level is obtained by applying the same method to the remaining 8 sub-squares. Using the same procedure, traditional Sierpinski and qaushi Sierpinski fractal structures could be obtained at different levels and in different geometries. 


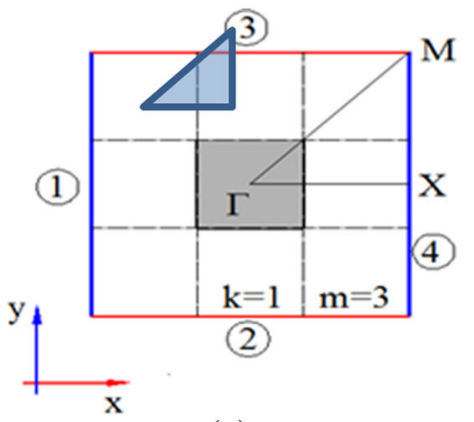

(a)

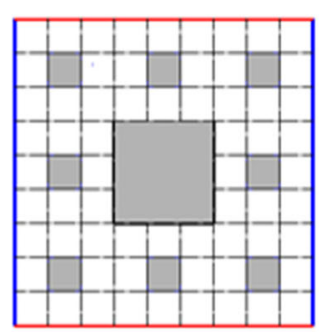

(b)

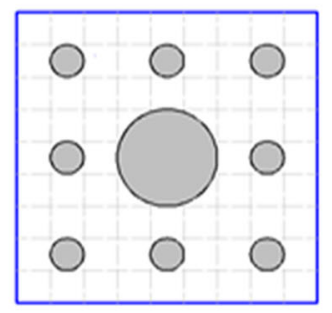

(d)

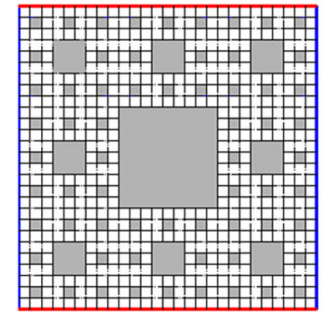

(c)

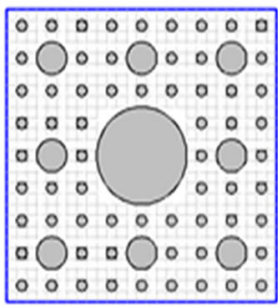

(e)

Figure 1. Traditional Sierpinski-carpet unit cells at different levels for square cross-section a) $L=1$ b) $\mathrm{L}=2 \mathrm{c}$ ) $\mathrm{L}=3$ for a circular cross-section d) $\mathrm{L}=2, \mathrm{e}) \mathrm{L}=3$.

The filling fraction, an important feature affecting the band structure in PnCs, was increased at every stage. In order to increase the filling fraction, some arrangements were made on the Sierpinski-carpet. For each stage, taking the initial frame (Figure 1a) as a reference, the unit cell was divided into grids and the super cells were obtained as depicted in Figure 1b-e.

The Floquet periodicity conditions were applied to the super cells and the band structures were obtained by using the finite element method (FEM) as seen in Figure 1a. In the Sierpinski-carpet fractal, the production procedure at different levels of the reference crosssections (square and circular cross-section) were used as seen in Figure 1. According to this production procedure, the grid number in the center of the unit cell for the value of $\mathrm{L}$ which equals to $i(i=2,3)$, the level of $n=m^{2 L}$ was obtained. Where $m$ is the number of grids in the $\mathrm{x}$ or $\mathrm{y}$ direction of step $1 . \mathrm{k}$ is the number of sub square $\mathrm{x}$ or $\mathrm{y}$ axis in the first stage. The mechanical properties of the rubber matrix are the density, $\rho=1300\left[\mathrm{~kg} / \mathrm{m}^{3}\right]$, elasticity $\mathrm{E}=1.175 \times 10^{-5}[\mathrm{GPa}]$, and Poisson ratio $v=0.4688$ respectively. The material properties of $\mathrm{LiTaO}_{3}$ are $\rho=7460\left[\mathrm{~kg} / \mathrm{m}^{3}\right], \mathrm{d}_{31}=-3.00 \times 10^{-12}[\mathrm{~m} / \mathrm{V}], \mathrm{d}_{33}=5.70 \times 10^{-12}[\mathrm{~m} / \mathrm{V}], \quad \mathrm{d}_{15}=$ $2.64 \times 10^{-12}[\mathrm{~m} / \mathrm{V}], \quad \mathrm{s}_{11}^{\mathrm{E}}=4.93 \times 10^{-12}\left[\mathrm{~m}^{2} \mathrm{~s}^{2} / \mathrm{kg}\right], \quad \mathrm{s}^{\mathrm{E}}{ }_{33}=4.32 \times 10^{-12}\left[\mathrm{~m} . \mathrm{s}^{2} / \mathrm{kg}\right], \quad \mathrm{s}_{12}^{\mathrm{E}}=$ $-5.19 \times 10^{-13}\left[\mathrm{~m} . \mathrm{s}^{2} / \mathrm{kg}\right], \quad \mathrm{s}_{13}^{\mathrm{E}}=-1.28 \times 10^{-12}\left[\mathrm{~m} . \mathrm{s}^{2} / \mathrm{kg}\right], \quad \mathrm{s}_{44}^{\mathrm{E}}=1.05 \times 10^{-11}\left[\mathrm{~m} . \mathrm{s}^{2} / \mathrm{kg}\right], \quad \mathrm{s}_{66}^{\mathrm{E}}=$ $1.09 \times 10^{-11}\left[\mathrm{~m} . \mathrm{s}^{2} / \mathrm{kg}\right], \varepsilon^{\mathrm{S}}{ }_{11} / \varepsilon_{\mathrm{o}}=53.5$, and $\varepsilon_{33}^{\mathrm{S}} / \varepsilon_{\mathrm{o}}=42.4[13,14]$.

\section{Discussions}

We used three types of cross-sections (as seen Figure 1) in square lattice Sierpinski carpet fractals with three levels (L1, L2 and L3) of PnC. 


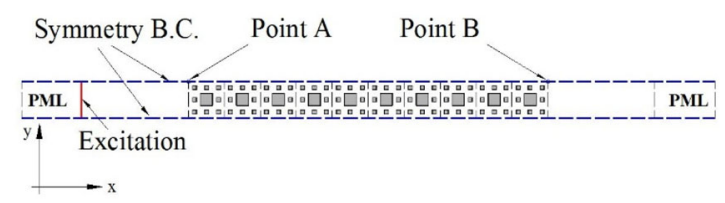

Figure 2. Band structure of quasi-Sierpinski carpet $\mathrm{PnC}$ consists of $\mathrm{LiTaO}_{3}$ in a rubber matrix in case of a K4, M6, L1 a) square rod b) circular rod.

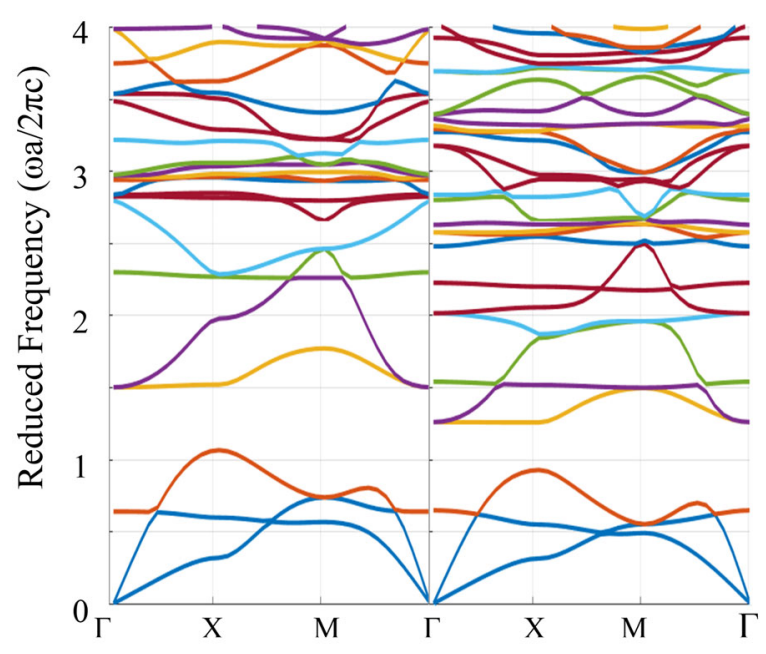

(a)

(b)

Figure 3. Topological phase in a band structure of $\mathrm{LiTaO}_{3} /$ rubber $\mathrm{PnC}$.

There was no significant band observed in any cross-section and or inclusion material in case of traditional Sierpinski-carpet L1, L2 and L3 levels. In quasi Sierpinski carpet where there was $\mathrm{K}=4$, and $\mathrm{M}=6 \mathrm{~L} 1$ level, there was a wide full band observed at a 33.99 gap size at 1.28, 30.23 gap size at 1.09 frequencies for square and circular $\mathrm{LiTaO}_{3}$ inclusions as seen in Figure 2.

In quasi-Sierpinki carpets with square cross-sections of $\mathrm{LiTaO}_{3} / \mathrm{Rubber} \mathrm{PnC}$ at $\mathrm{L} 2$ level, low-frequency bands observed at L1 level disappeared, but the high-frequency bands with gap sizes of $4.92,7.78,5.37,4.01,3.98$, and 8.55 occurred at reduced frequencies $2.90,4.07,5.46,5.76,6.13$ and 7.48 (Table 2).

In a circular cross-section $\mathrm{LiTaO}_{3} /$ rubber quasi-Sierpinski carpet PnC at $\mathrm{L} 2$ level, the low-frequency bands observed at the L1 level disappeared, but a lot of high-frequency bands occurred at 1.89 and 1.43 gap sizes with frequencies of 3.70 and 4.57 .

Figure 3 shows the dispersion relation of $\mathrm{LiTaO}_{3} /$ rubber $\mathrm{PnC}$ with $\mathrm{M} 3 \mathrm{~K} 1$ level $1 \mathrm{PnC}$ with square cross-section inclusion. It can be seen that $\mathrm{LiTaO}_{3}$ based $\mathrm{PnC}$ has a topological phase as in some narrow-band insulators. The band structure contains Dirac point degeneracy and the resulting acoustic bands have nonzero Chern numbers, indicating that they are topologically nontrivial [15].

Figure 4 shows the $3 \mathrm{D}$ band structure of $\mathrm{LiTaO}_{3} /$ rubber $\mathrm{PnC}$ for the ground and second mode with their associated reduced velocities along the $\Gamma-\mathrm{X}-\mathrm{M}-\Gamma$ direction. 

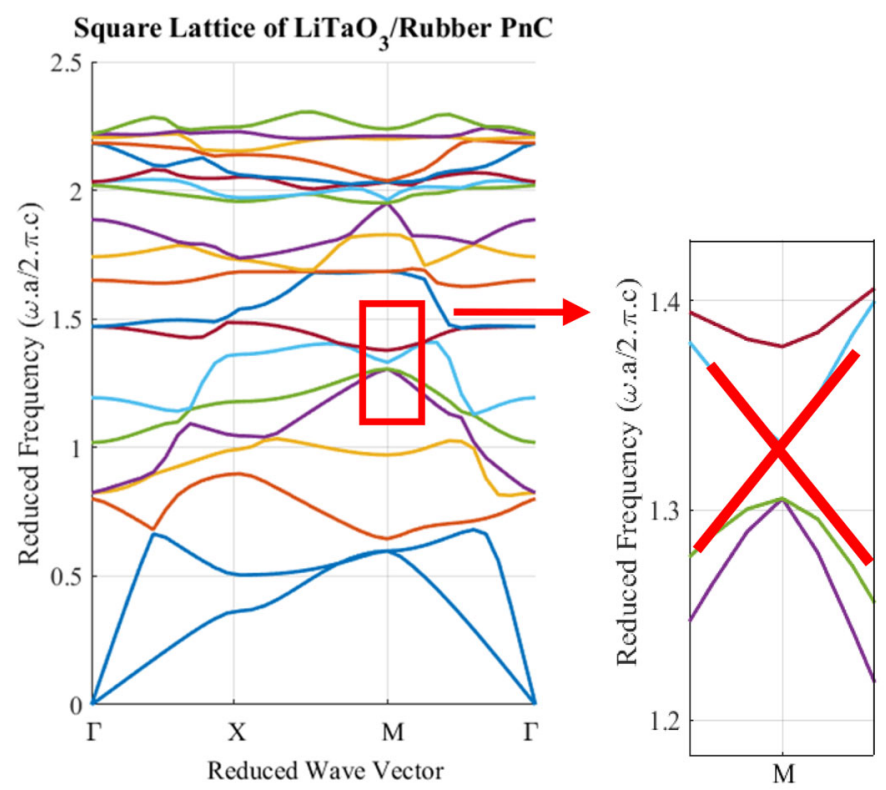

Figure 4. 3D Band structure of $\mathrm{LiTaO}_{3} /$ rubber PnC for the first two bands.

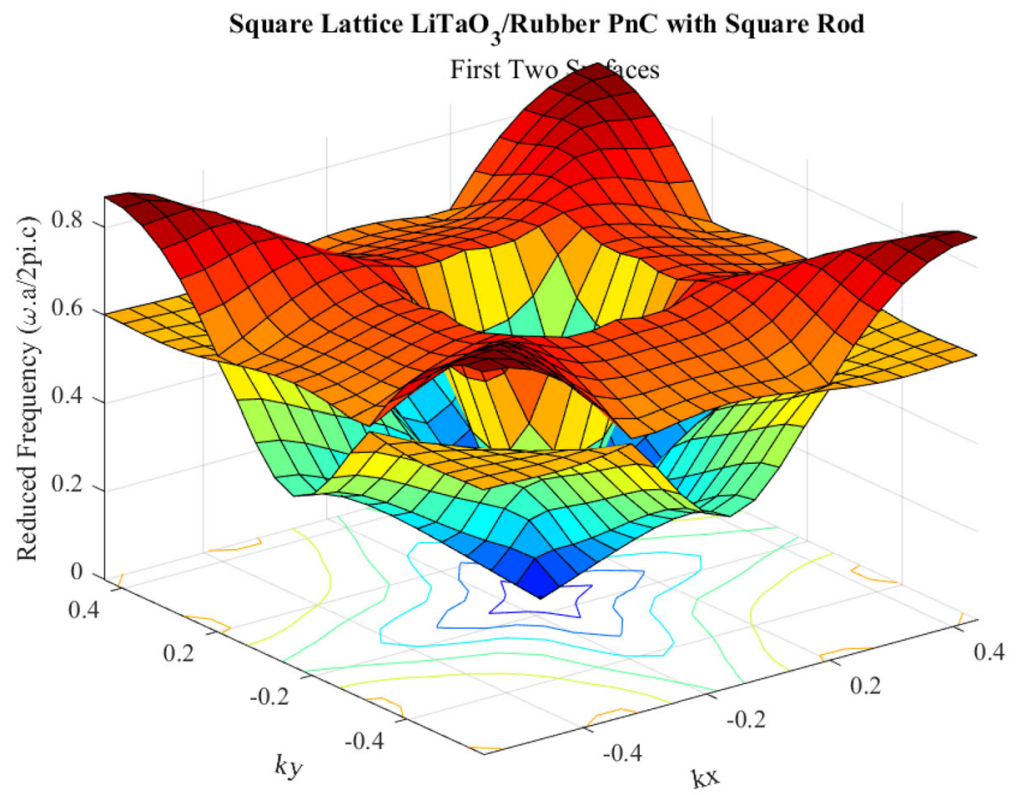

Figure 5. Finite array of scatters for calculating the transmission loss of PnC.

We obtained the transmission spectra of $\mathrm{PnC}$ by using the finite array in Figure 5 and compared them with the band structure of case M6K4. Figure 6 shows a comparison of the band structure and transmission spectra for all of investigated types scatterers.

As seen in Figure 6, there was a narrow band observed at reduced frequencies between 1.28 and 1.38. On the other hand a wide band was observed between 1.71 and 2.11 as well as 1.74 and 3.16. 


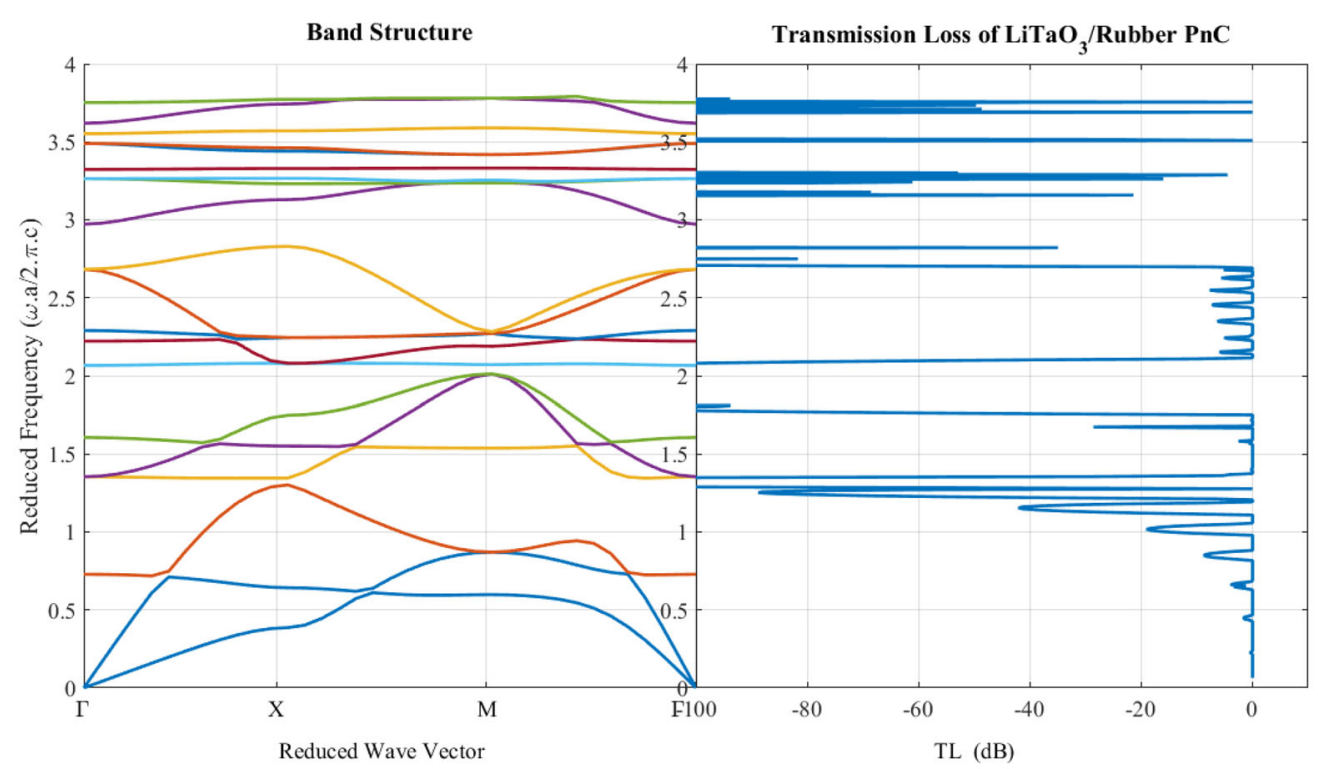

Figure 6. Band structure of square cross-section scatterers $\mathrm{LiTaO}_{3}$ in a rubber matrix M6K4 $\mathrm{L} 2$ level $\mathrm{PnC}$ and the transmissions spectra.

Table 1. Full band gap size variation of a quasi-Sierpinski carpet case M6K4 Level $1 \mathrm{LiTaO}_{3} /$ rubber $\mathrm{PnC}$ with a square and circular cross-section.

\begin{tabular}{|c|c|c|c|c|}
\hline & \multicolumn{2}{|c|}{ Square cross-section } & \multicolumn{2}{|c|}{ Circular cross-section } \\
\hline & Mid Gap (a/c) & Gap Size (\%) & Mid Gap (a/c) & Gap Size (\%) \\
\hline 1. Band & 1.28 & 34.00 & 1.09 & 30.23 \\
\hline 2. Band & 3.10 & 0.32 & 1.52 & 0.01 \\
\hline 3. Band & - & - & 2.18 & 0.15 \\
\hline 4. Band & - & - & 3.38 & 0.83 \\
\hline 5. Band & - & - & 3.73 & 0.33 \\
\hline 6. Band & - & - & 4.16 & 0.05 \\
\hline
\end{tabular}

Table 2. Some full band gap size variation of a quasi-Sierpinski carpet case M6K4 Level $2 \mathrm{LiTaO}_{3} /$ rubber PnC with a square and circular cross-section.

\begin{tabular}{|c|c|c|c|c|}
\hline & \multicolumn{2}{|c|}{ Square cross-section } & \multicolumn{2}{|c|}{ Circular cross-section } \\
\hline & Mid Gap (a/c) & Gap Size (\%) & Mid Gap (a/c) & Gap Size (\%) \\
\hline 1. Band & 1.32 & 3.26 & 2.45 & 0.12 \\
\hline 2. Band & 2.04 & 2.65 & 2.53 & 0.61 \\
\hline 3. Band & 2.90 & 4.92 & 2.62 & 0.24 \\
\hline 4. Band & 3.38 & 2.55 & 3.57 & 0.03 \\
\hline 5. Band & 4.07 & 7.78 & 3.70 & 1.89 \\
\hline 6. Band & 5.19 & 3.44 & 4.24 & 0.28 \\
\hline 7. Band & 5.46 & 5.37 & 4.58 & 1.44 \\
\hline 8. Band & 5.76 & 4.01 & 5.87 & 2.70 \\
\hline 9. Band & 6.13 & 3.98 & 6.02 & 0.83 \\
\hline 10. Band & 7.48 & 8.55 & 6.98 & 0.76 \\
\hline
\end{tabular}

The band structures of a quasi-Sierpinski carpet PnCs consisting of various piezoelectric inclusions with square cross-sections at L1, L2 and L3 levels are shown in Tables 1-3. 
Table 3. Some full band gap size variation of the quasi-Sierpinski carpet case $\mathrm{M} 6 \mathrm{~K} 4$ Level $3 \mathrm{LiTaO}_{3} /$ rubber PnC with a square and circular cross-section.

\begin{tabular}{|c|c|c|c|c|}
\hline & \multicolumn{2}{|c|}{ Square cross-section } & \multicolumn{2}{|c|}{ Circular cross-section } \\
\hline & Mid Gap $(a / c)$ & Gap Size (\%) & Mid Gap (a/c) & Gap Size (\%) \\
\hline 1. Band & 1.42 & 3.30 & 1.65 & 5.54 \\
\hline 2. Band & 2.14 & 8.23 & 1.84 & 0.04 \\
\hline 3. Band & 2.99 & 7.12 & 2.17 & 3.27 \\
\hline 4. Band & 3.29 & 1.51 & 2.34 & 6.05 \\
\hline 5. Band & 3.43 & 4.86 & 2.45 & 0.05 \\
\hline 6. Band & 3.58 & 2.40 & 2.54 & 2.61 \\
\hline 7. Band & 3.75 & 1.78 & 2.93 & 1.88 \\
\hline 8. Band & 3.92 & 3.41 & 3.03 & 0.77 \\
\hline
\end{tabular}

As can be seen from the tables, Level 1 (L1) PnC has a wide band range at low frequencies, has no multiband capability and L2 and L3 level PnCs have multiband characteristics at high frequencies, while the low frequency band disappears.

\section{Conclusion}

In this study, the band gap structure and transmission in two dimensional $\mathrm{LiNbO}_{3}$ based Sierpinski carpet phononic crystal with triangular and circular cross-sections piezoelectric scatterers in a rubber matrix were investigated based on finite element simulation. We can summarize the results as follows:

- $\quad$ The proposed PnC has topological insulator properties.

- $\mathrm{LiTaO}_{3}$ based circular cross-section PnC has a gap at lower frequency but square cross-section $\mathrm{PnC}$ has more bands at both lower and higher frequencies.

- As seen in Figure 2, Level 1 (L1) PnC does not have multiband properties, although a multiband exists at the L2 and L3 levels, L3 has difficulty in production and the width of the new bands is narrower than the L2 level and is not suitable for practical applications as seen in Tables 2 and 3.

- As shown in [16] by applying voltage to the piezoelectric inclusions the proposed $\mathrm{PnC}$ can be used as actively guiding waves.

- New research areas will emerge in the development of various devices in the areas of RF communication, sensor, medical ultrasound, acoustic filter and wave guiding.

\section{References}

[1] H. Oraizi, and S. Hedayati, Miniaturized UWB monopole microstrip antenna design by the combination of giusepe peano and sierpinski carpet fractals, Antennas Wirel. Propag. Lett. 10, 67 (2011). DOI: 10.1109/LAWP.2011.2109030.

[2] S. Tarafdar et al., Modelling porous structures by repeated Sierpinski carpets, Physica A 292 (1-4), 1 (2001). DOI: 10.1016/S0378-4371(00)00573-2.

[3] W. Steurer, and D. Sutter-Widmer, Photonic and phononic quasicrystals, J. Phys. D: Appl. Phys. 40 (13), R229 (2007). DOI: 10.1088/0022-3727/40/13/R01.

[4] R. C. Norris, J. S. Hamel, and P. Nadeau, Phononic band gap crystals with periodic fractal inclusions: theoretical study using numerical analysis, J.Appl. Phys. 103 (10), 104908 (2008). DOI: 10.1063/1.2931955. 
[5] F. De Nicola et al., Multiband plasmonic sierpinski carpet fractal antennas, ACS Photonics. 5 (6), 2418 (2018). DOI: 10.1021/acsphotonics.8b00186.

[6] N.-K. Kuo, and G. Piazza, Fractal phononic crystals in aluminum nitride: An approach to ultra-high frequency bandgaps, Appl. Phys. Lett. 99 (16), 163501 (2011). DOI: 10.1063/1. 3651760 .

[7] S. Castineira-Ibanez et al., Design, manufacture and characterization of an acoustic barrier made of multi-phenomena cylindrical scatterers arranged in a fractal-based geometry, Arch. Acoust. 37 (4), 455 (2012). DOI: 10.2478/v10168-012-0057-9.

[8] L. Xiao-Jian, and F. You-Hua, Band structure characteristics of T-square fractal phononic crystals, Chin. Phys. B 22 (3), 036101 (2013). DOI: 10.1088/1674-1056/22/3/036101.

[9] S. Castiñeira-Ibáñez et al., Quantitative characterization of bandgap properties of sets of isolated acoustic scatterers arranged using fractal geometries, Appl. Phys. Express 7 (4), 042201 (2014). DOI: 10.7567/APEX.7.042201.

[10] K. Wang, Y. Liu, and T. Liang, Band structures in Sierpinski triangle fractal porous phononic crystals, Physica B 498, 33 (2016). DOI: 10.1016/j.physb.2016.06.018.

[11] J. Huang, Z. Shi, and W. Huang, Multiple band gaps of phononic crystals with quasiSierpinski carpet unit cells, Physica B 516, 48 (2017). DOI: 10.1016/j.physb.2017.04.022.

[12] K. Wang et al., Band structures in fractal grading porous phononic crystals, J. Phys. Chem. Solids 116, 367 (2018). DOI: 10.1016/j.jpcs.2018.01.048.

[13] https://www.efunda.com/materials/piezo/material_data/matdata_output.cfm?Material_ID= LiTaO (accessed Apr. 11, 2019).

[14] T. Yamada, H. Iwasaki, and N. Niizeki, Piezoelectric and elastic properties of $\mathrm{LiTaO}_{3}$ : temperature characteristics, Jpn. J. Appl. Phys. 8 (9), 1127 (1969). DOI: 10.1143/JJAP.8.1127.

[15] F. Marcel, and L. Molenkamp, Topological insulators, 1st ed. (Elsevier Science, Burlington, 2013), Vol. 6.

[16] J. H. Oh et al., Active wave-guiding of piezoelectric phononic crystals, Appl. Phys. Lett. 99, 083505 (2011). DOI: 10.1063/1.3630231. 